\title{
The Existence of a Polynomial Inverse Integrating Factors and Studies About the Limit Cycles for Cubic, Quartic and Quintic Polynomial Systems
}

\author{
Ahmed Muhammad Hussein ${ }^{1} \quad$ Sadeq Taha Abdulazeez ${ }^{2^{*}}$
}

${ }^{1}$ Department of Mathematics, College of Science, University of Duhok, Duhok, Iraq.

${ }^{2}$ Department of Mathematics, College of Basic Education, University of Duhok, Duhok, Iraq.

"Corresponding author: ahmad.husien@uod.ac' sadeq.taha@uod.ac*

"ORCID ID: https://orcid.org/0000-0002-1598-5397, https://orcid.org/0000-0003-4515-1585*

Received 30/7/2019, Accepted 8/9/2020, Published Online First 11/1/2021, Published 1/6/2021

\section{Abstract:}

The main aims of our study are the presence of reverse polynomial integration factors and studies about the limit cycles for real polynomial in two-dimensional autonomous system of the form

$$
\begin{aligned}
& \dot{\alpha}=\sum_{j=0}^{m} \sum_{i=0}^{j} a_{i, j-i} \alpha^{i} \beta^{j-i}=P_{m}(\alpha, \beta) \\
& \dot{\beta}=\sum_{j=0}^{m} \sum_{i=0}^{j} b_{i, j-i} \alpha^{i} \beta^{j-i}=Q_{m}(\alpha, \beta)
\end{aligned}
$$

Where $P_{m}(\alpha, \beta)$ and $Q_{m}(\alpha, \beta)$ are polynomials of degree $(m), m=3,4$ and 5 .

Key words: Limit cycles, Polynomials Inverse integrating factors, Polynomial systems.

\section{Introduction:}

Some results of polynomial inverse integrating factor and study about limit cycle of system (1) are obtained, there are many works achieved on the two-dimensional autonomous system, According to polynomial inverse integrating factor and limit cycles, readers can judge Andronov(1), Ferragut (2), García et al.(3), Giné \& Llibre (4, 5), García \& Grau (6), Coll et al. (7), Perko(8), Poincaré (9), Van der Pol (10) and YanQian(11).

Our project is structured as follows and consists of two sections:

The first section, is an introduction to planar differential systems. The most basic definitions and results of general type are also given, including the main topics discussed in this paper. The method must be lead to find polynomials inverse integrating factor, and some results about the existence of inverse polynomial integrating factors of system (1) are given in section two.

Introduction to planar differential systems (3-6): Firstly, the following systems of form are concerned with independent polynomial planar differential

$$
\begin{aligned}
& \dot{\alpha}=P(\alpha, \beta), \\
& \dot{\beta}=Q(\alpha, \beta),
\end{aligned}
$$

where $P(\alpha, \beta)$ and $Q(\alpha ; \beta)$ are continuously partial first order derivative functions of class $U$ and $U \subseteq \mathbb{R}^{2}$, secondly, restrict $P(\alpha, \beta)$ and $Q(\alpha, \beta)$ to be elements in a real polynomial loop in two variables $\mathbb{R}[\alpha, \beta]$. The point indicates the derivation of the independent variable $\mathrm{t}$, which is usually called time, that is $\dot{\alpha}=\frac{d \alpha}{d t}$. The vector field linked to system (1.1) will be denoted as

$$
\chi(\alpha, \beta)=P(\alpha, \beta) \frac{\partial}{\partial \alpha}+Q(\alpha, \beta) \frac{\partial}{\partial \beta} .
$$

\section{Definition 1 (3):}

Define the divergence of a vector field $\chi$, written $\operatorname{div} \chi$ or $\nabla \cdot \chi$, as the dot product of del with $\chi$. So if the vector $\chi=\langle P, Q\rangle$, then

$$
\operatorname{div} \chi=\nabla \cdot \chi=\left\langle\frac{\partial}{\partial \alpha}, \frac{\partial}{\partial \beta}\right\rangle \cdot\langle P, Q\rangle=\frac{\partial P}{\partial \alpha}+\frac{\partial Q}{\partial \beta} .
$$

Notice that $\operatorname{div} \chi$ is a scalar.

\section{Definition 2 (12):}

The nonzero function $V: U \rightarrow R$ is said to be the inverse integral factor of (1.1) for category 
$C^{1}(\mathrm{U}, \mathrm{R})$, and is not local empty and fulfill the following linear PDEs:

$$
P \frac{\partial V}{\partial \alpha}+Q \frac{\partial V}{\partial \beta}=\left(\frac{\partial P}{\partial \alpha}+\frac{\partial Q}{\partial \beta}\right) V .
$$

In short, the reverse integration factor of $V(\alpha, \beta)$ system (1.1) fulfill

$$
\chi V=V \operatorname{div} \chi .
$$

The inverse integration factor is a significant material in our study.

Theorem 1 (3),(13):

Let $V_{1}, \ldots, V_{p}$ be inverse integration factors of (1.1) and $a_{1}, \ldots, a_{p} \in \mathbb{R}$. Then, the function $V=$ $\sum_{i=1}^{p} a_{i} V_{i}$ is inverse integration factor of (1.1).

Definition 3 (4),(15):

The singular point $\left(\alpha_{0}, \beta_{0}\right)$ of (1.1) is said to be center if there is a neighborhood $N$ of $\left(\alpha_{0}, \beta_{0}\right)$ such that all the trajectories of $N \backslash\left\{\left(\alpha_{0}, \beta_{0}\right)\right\}$ are periodic.

\section{Definition 4 (5), (16-17):}

If $\mathrm{C}$ is an isolated closed curve, then $\mathrm{C}$ is called a limit cycle. The important relationship between the limit cycle and the inverse integrating factor, verified in (1) and (14).

Theorem 2 (2), (6):

Let $V: U \rightarrow R$ be the reverse merge factor of (1.1). If $\rho \subset \mathrm{U}$ is a boundary cycle of (1.1), then $\rho$ is included in group.

$$
\Sigma=\{(\alpha, \beta) \in U: V(\alpha, \beta)=0\} .
$$

Inverse Integrating Factors for polynomial systems (4), (6):

This portion is concentrated on some polynomial results of the inverse integral factors of cubic, quartic and quintic polynomial systems.

Firstly, systems are considered real polynomial differential systems (1.1) of the degree $m=3,4,5$. Our aims are to obtain the polynomial inverse integral factor $V(\alpha, \beta)$.

Method for calculating inverse polynomial integrating factors for systems (2), (6), (14):

Consider the real planar systems (1), suppose that $P_{m}$ and $Q_{m}$ have no intersection factors; in general to find the inverse integrating factors use equation (1.2).

Method 1. Look for real inverse polynomial integrals factor of degree $\mathrm{k} \in \mathrm{N}$, and write $V(\alpha, \beta)$ as

$$
V(\alpha, \beta)=\sum_{i+j=0}^{k} V_{i, j} \alpha^{i} \beta^{j},
$$

Where $V_{i, j} \in \mathrm{R}$. equation (1.2) is a polynomial equation, because $P_{m}(\alpha, \beta), Q_{m}(\alpha, \beta)$ and $V$ are Polynomial functions which can be written as aligner system with an unknown $V_{i, j}, i+j=$ $o, \ldots, k$.

\section{Theorem 3 (12):}

Let $V(\alpha, \beta)$ be the inverse polynomial inverse factor to incorporate the degree of $\mathrm{k}$ into the system (1). Then equation (1.2) is equal in value to a linear homo system:

$$
A_{\mathrm{k}} V^{\mathrm{k}}=\mathrm{o},
$$

Were $A_{k}$ is matrix and

$V^{k}=$

$\left(\mathrm{V}_{0,0}, \mathrm{~V}_{1,0}, \mathrm{~V}_{0,1}, \mathrm{~V}_{2,0}, \mathrm{~V}_{1,1}, \mathrm{~V}_{0,2}, \ldots, V_{k, 0}, V_{k-1,1}, \ldots, V_{1, k-1}, V_{0, k}\right)^{\mathrm{T}}$

$V^{k}$ is vector of coefficient for $V(\alpha, \beta)$.

The presence of an inverse integrals factor for cubic polynomial systems

\section{Theorem 4}

The nonlinear systems

$$
\begin{aligned}
& \dot{\alpha}=-\beta-3 \alpha^{2} \beta \\
& \dot{\beta}=\alpha-9 \alpha \beta^{2}
\end{aligned}
$$

have an inverse integral factor

$$
\begin{gathered}
V(\alpha, \beta)=v_{0,0}\left(1+12 \alpha^{2}+54 \alpha^{4}+108 \alpha^{6}\right. \\
\left.+81 \alpha^{8}\right) .
\end{gathered}
$$

Proof: let $V(\alpha, \beta)$ be the inverse integral factor so by (Definition 2) and equation (1.2), it must be the following system

$$
\begin{gathered}
\mathrm{P} \frac{\partial V}{\partial \alpha}+Q \frac{\partial V}{\partial \beta}=\left(\frac{\partial \mathrm{P}}{\partial \alpha}+\frac{\partial Q}{\partial \beta}\right) V \\
\left(-\beta-3 \alpha^{2} \beta\right) \frac{\partial V}{\partial \alpha}+\left(\alpha-9 \alpha \beta^{2}\right) \frac{\partial V}{\partial \beta} \\
=(-24 \alpha \beta) V
\end{gathered}
$$

Solve our semi-linear equation (2.3), the result would be

$$
\frac{d \alpha}{-\beta-3 \alpha^{2} \beta}=\frac{d \beta}{\alpha-9 \alpha \beta^{2}}=\frac{d V}{(-24 \alpha \beta) V},
$$

in general $V(\alpha, \beta)$ is extremely difficult to solve. Thus, the method is ignored Eq. 2.3. So by the Method, $V(\alpha, \beta)=\sum_{i+j=0}^{k} V_{i, j} \alpha^{i} \beta^{j}$, apply for $k=8$, it gives us

$$
\begin{gathered}
V(\alpha, \beta)=v_{0,0}+v_{2,0} \alpha^{2}+v_{4,0} \alpha^{4}+v_{6,0} \alpha^{6} \\
+v_{8,0} \alpha^{8} \\
\left(-\beta-3 \alpha^{2} \beta\right) \frac{\partial V}{\partial \alpha}+\left(\alpha-9 \alpha \beta^{2}\right) \frac{\partial V}{\partial \beta} \\
=(-24 \alpha \beta) V \\
\left(-\beta-3 \alpha^{2} \beta\right)\left(2 v_{2,0} \alpha+4 v_{4,0} \alpha^{3}+6 v_{6,0} \alpha^{5}\right. \\
\left.+8 v_{8,0} \alpha^{7}\right) \\
=(-24 \alpha \beta)\left(v_{0,0}+v_{2,0} \alpha^{2}\right. \\
\left.+v_{4,0} \alpha^{4}+v_{6,0} \alpha^{6}+v_{8,0} \alpha^{8}\right)
\end{gathered}
$$

The coefficients of $\alpha^{5} \beta, \alpha^{9} \beta, \alpha^{7} \beta, \alpha^{3} \beta$ and $\alpha \beta$ are:

$$
\begin{aligned}
& -12 v_{0,0}+v_{2,0}=0 \\
& -9 v_{2,0}+2 v_{4,0}=0 \\
& -2 v_{4,0}+v_{6,0}=0 \\
& -3 v_{6,0}+4 v_{8,0}=0
\end{aligned}
$$

The above system will give 


$$
\begin{gathered}
\left(\begin{array}{ccccc}
-12 & 1 & 0 & 0 & 0 \\
0 & -9 & 2 & 0 & 0 \\
0 & 0 & -2 & 1 & 0 \\
0 & 0 & 0 & -3 & 4 \\
0 & 0 & 0 & 0 & 0
\end{array}\right)\left(\begin{array}{l}
v_{0,0} \\
v_{2,0} \\
v_{4,0} \\
v_{6,0} \\
v_{8,0}
\end{array}\right)=0 \\
A_{5} V^{5}=0,
\end{gathered}
$$

After solving the system

$v_{4,0}=54 v_{0,0}, \quad v_{2,0}=12 v_{0,0}, \quad v_{6,0}=108 v_{0,0}$

and $v_{8,0}=81 v_{0,0}$

$v_{0,0}$ is an independent constant.

Substitute $v_{2,0}, v_{4,0}, v_{6,0}$ and $v_{8,0}$ in $V(\alpha, \beta)$

$$
\begin{gathered}
V(\alpha, \beta)=v_{0,0}\left(1+12 \alpha^{2}+54 \alpha^{4}+108 \alpha^{6}\right. \\
\left.+81 \alpha^{8}\right) .
\end{gathered}
$$

\section{Corollary 1}

Proof: similar to (Theorem 4) after substitute in $V(\alpha, \beta)$, get the system

$$
\begin{gathered}
\left(\begin{array}{ccc}
4 & -1 & 0 \\
0 & 2 & -2 \\
0 & -1 & 1
\end{array}\right)\left(\begin{array}{l}
\mathrm{v}_{0,0} \\
\mathrm{v}_{1,1} \\
\mathrm{v}_{2,2}
\end{array}\right)=0 . \\
A_{3} V^{3}=0,
\end{gathered}
$$

After solving the system

$v_{1,1}=4 v_{0,0}$ and , $v_{2,2}=4 v_{0,0}$

$\mathrm{v}_{0,0}$ is an independent constant.

Substitute $v_{1,1}$ and $v_{2,2}$ in $V(\alpha, \beta)$

\section{Theorem 5}

$$
V(\alpha, \beta)=v_{0,0}\left(1+4 \alpha \beta+4 \alpha^{2} \beta^{2}\right) \text {. }
$$

The Cubic systems in all the previous corollaries cannot contain limit cycles.

Proof: To prove that, it is followed straight by (Theorem 2) since the illustration of reverse integrals factors is not associated with convex functions.

The presence of inverse integrals factors for Quartic polynomial systems

\section{Theorem 6}

The nonlinear system

Proof: similar to the above theorem, after substituting in $V(\alpha, \beta)$, the following system can be given

$$
\begin{gathered}
12 v_{0,0}+v_{2,0}=0 \\
9 v_{2,0}+2 v_{4,0}=0 \\
2 v_{4,0}+v_{6,0}=0 \\
3 v_{6,0}+4 v_{8,0}=0 \\
\left(\begin{array}{ccccc}
12 & 1 & 0 & 0 & 0 \\
0 & 9 & 2 & 0 & 0 \\
0 & 0 & 2 & 1 & 0 \\
0 & 0 & 0 & 3 & 4 \\
0 & 0 & 0 & 0 & 0
\end{array}\right)\left(\begin{array}{l}
v_{0,0} \\
v_{2,0} \\
v_{4,0} \\
v_{6,0} \\
v_{8,0}
\end{array}\right)=0 \\
A_{5} V^{5}=0,
\end{gathered}
$$

After solving the system

$$
\begin{gathered}
a_{1,0} \alpha+a_{0,1} \beta+a_{2,0} \alpha^{2}+a_{1,1} \alpha \beta+a_{3,0} \alpha^{3} \\
\dot{\alpha}=+a_{2,1} \alpha^{2} \beta+a_{1,2} \alpha \beta^{2}+a_{0,3} \beta^{3}+a_{4,0} \alpha^{4} \\
+a_{3,1} \alpha^{3} \beta+a_{1,3} \alpha \beta^{3}+a_{2,2} \alpha^{2} \beta^{2}, \\
\dot{\beta} \quad \\
\dot{\beta} \quad \\
b_{1,0} \alpha+b_{0,1} \beta+b_{2,0} \alpha^{2}+b_{1,1} \alpha \beta+b_{0,2} \beta^{2} \\
+b_{3,0} \alpha^{3}+b_{2,1} \beta \alpha^{2}+b_{1,2} \beta^{2} \alpha+b_{0,3} \beta^{3}+b_{4,0} \alpha^{4} \\
+b_{3,1} \beta \alpha^{3}+b_{1,3} \alpha \beta^{3}+b_{2,2} \alpha^{2} \beta^{2}+b_{0,4} \beta^{4},
\end{gathered}
$$

has an inverse integrating factor

$$
\begin{aligned}
V(\alpha, \beta) & =v_{2,0} \alpha^{2}+v_{0,2} \beta^{2}+v_{3,0} \alpha^{3} \\
& +v_{1,2} \alpha \beta^{2}+v_{4,0} \alpha^{4}+v_{2,2} \alpha^{2} \beta^{2} \\
& +v_{0,4} \beta^{4}+v_{5,0} \alpha^{5}+v_{1,4} \alpha \beta^{4} \\
& +v_{3,2} \alpha^{3} \beta^{2} .
\end{aligned}
$$

find the optimal solutions of the inverse integrating factor.

Proof: let $V(\alpha, \beta)$ be the inverse integrating factor so by (Definition 2) and equation (1.2), the result must be given as below

$$
\mathrm{P} \frac{\partial V}{\partial \alpha}+\mathrm{Q} \frac{\partial V}{\partial \beta}=\left(\frac{\partial P}{\partial \alpha}+\frac{\partial Q}{\partial \beta}\right) V
$$

After substituting $P$ and $Q$, quasi linear equation is given.

In general, it is extremely difficult to solve quasi linear equation. Thus, the method is ignored. $V(\alpha, \beta)=\sum_{i+j=0}^{k} V_{i, j} \alpha^{i} \beta^{j}$, apply for $k=5$, so the result is supported by the method

$$
\begin{gathered}
V(\alpha, \beta)=v_{2,0} \alpha^{2}+v_{0,2} \beta^{2}+v_{3,0} \alpha^{3}+v_{1,2} \alpha \beta^{2}+v_{4,0} \alpha^{4}+v_{2,2} \alpha^{2} \beta^{2}+v_{0,4} \beta^{4}+v_{5,0} \alpha^{5}+v_{1,4} \alpha \beta^{4} \\
+v_{3,2} \alpha^{3} \beta^{2}
\end{gathered}
$$




$$
\begin{aligned}
& \left(a_{1.0} \alpha+a_{0.1} \beta+a_{2.0} \alpha^{2}+a_{1.1} \alpha \beta+a_{3.0} \alpha^{3}+a_{2.1} \alpha^{2} \beta+a_{1.2} \alpha \beta^{2}+a_{0.3} \beta^{3}+a_{4.0} \alpha^{4}+a_{3.1} \alpha^{3} \beta\right. \\
& \left.+a_{1.3} \alpha \beta^{3}+a_{2.2} \alpha^{2} \beta^{2}\right) \frac{\partial V}{\partial \alpha} \\
& +\left(b_{1.0} \alpha+b_{0.1} \beta+b_{2.0} \alpha^{2}+b_{1.1} \alpha \beta+b_{0.2} \beta^{2}+b_{3.0} \alpha^{3}+b_{2.1} \beta \alpha^{2}+b_{1.2} \beta^{2} \alpha+b_{0.3} \beta^{3}\right. \\
& \left.+b_{4.0} \alpha^{4}+b_{3.1} \beta \alpha^{3}+b_{1.3} \alpha \beta^{3}+b_{2.2} \alpha^{2} \beta^{2}+b_{0.4} \beta^{4}\right) \frac{\partial V}{\partial \beta} \\
& =\left(a_{1.0}+2 a_{2.0} \alpha+a_{1.1} \beta+3 a_{3.0} \alpha^{2}+2 a_{2.1} \alpha \beta+a_{1.2} \beta^{2}+4 a_{4.0} \alpha^{3}+3 a_{3.1} \alpha^{2} \beta+a_{1.3} \beta^{3}+2 a_{2.2} \alpha \beta^{2}\right. \\
& +b_{0.1}+b_{1.1} \alpha+2 b_{0.2} \beta+b_{2.1} \alpha^{2}+2 b_{1.2} \beta \alpha+3 b_{0.3} \beta^{2}+b_{3.1} \alpha^{3}+3 b_{1.3} \alpha \beta^{2}+2 b_{2.2} \alpha^{2} \beta \\
& \left.+4 b_{0.4} \beta^{3}\right) V
\end{aligned}
$$

After calculating, the coefficients of terms are:

$$
\begin{gathered}
\left(-2 a_{2,0}+b_{1,1}\right) v_{0,2}+b_{0,1} v_{1,2}=0 \\
2 b_{2,0} v_{1,2}-2 b_{1,2} v_{2,0}+\left(2 a_{1,1}-2 b_{0,2}\right) v_{3,0}+2 b_{3,0} v_{0,2}+2 b_{1,0} v_{2,2}+4 a_{0,1} v_{4,0}=0 \\
\left(a_{1,1}-2 b_{0,2}\right) v_{2,0}+2 b_{2,0} v_{0,2}+2 b_{1,0} v_{1,2}+3 a_{0,1} v_{3,0}=0 \\
2 a_{0,3} v_{2,0}-2 a_{2,1} v_{0,2}+2 a_{0,1} v_{2,2}+4 b_{1,0} v_{0,4}=0 \\
-3 a_{3,0} v_{0,2}+b_{0,1} v_{2,2}+b_{1,1} v_{1,2}+a_{1,2} v_{2,0}+a_{1,0} v_{2,2}-a_{2,0} v_{1,2}+b_{2,1} v_{0,2}-3 b_{0,3} v_{2,0}=0 \\
2 b_{1,0} v_{0,2}+2 a_{0,1} v_{2,0}=0 \\
2 a_{1,0} v_{3,0}-b_{0,1} v_{3,0}-b_{1,1} v_{2,0}=0 \\
a_{1,0} v_{2,0}-b_{0,1} v_{2,0}=0 \\
a_{0,1} v_{1,2}-a_{1,1} v_{0,2}=0 \\
b_{0,1} v_{0,2}-a_{1,0} v_{0,2}=0 \\
-b_{2,1} v_{2,0}+3 a_{1,0} v_{4,0}-b_{0,0} v_{4,0}+a_{2,0} v_{3,0}-a_{3,0} v_{2,0}-b_{1,1} v_{3,0}=0 \\
-b_{0,3} v_{0,2}+3 b_{0,1} v_{0,4}-a_{1,2} v_{0,2}-2 a_{1,0} v_{0,4}=0
\end{gathered}
$$

$-3 b_{0,3} v_{3,0}+b_{3,1} v_{0,2}+b_{1,1} v_{2,2}+2 a_{1,2} v_{3,0}+b_{2,1} v_{1,2}-4 a_{4,0} v_{0,2}+b_{0,1} v_{3,2}+2 a_{1,0} v_{3,2}-2 a_{3,0} v_{1,2}$$$
-3 b_{1,3} v_{2.0}=0
$$$$
-b_{0,3} v_{1,2}+3 b_{1,1} v_{0,4}+3 b_{0,1} v_{1,4}-2 a_{2,0} v_{0,4}-b_{1,3} v_{0,2}-2 a_{2,2} v_{0,2}=0
$$$$
-b_{0,1} v_{5,0}-2 a_{4,0} v_{2,0}-b_{3,1} v_{2,0}-b_{2,1} v_{3,0}+2 a_{2,0} v_{4,0}-b_{1,1} v_{4,0}+4 a_{1,0} v_{5,0}=0
$$$$
b_{0,3} v_{0,4}-a_{1,2} v_{0,4}=0
$$$$
a_{0,3} v_{1,4}-a_{1,3} v_{0,4}=0
$$$$
-2 b_{0,4} v_{0,2}+a_{0,3} v_{1,2}-a_{1,3} v_{0,2}-a_{1,1} v_{0,4}+a_{0,1} v_{1,4}+2 b_{0,2} v_{0,4}=0
$$$$
a_{3,0} v_{4,0}-b_{1,1} v_{5,0}-b_{3,1} v_{3,0}-a_{4,0} v_{3,0}+3 a_{2,0} v_{5,0}-b_{2,1} v_{4,0}=0
$$$$
a_{4,0} v_{5,0}-b_{3,1} v_{5,0}=0
$$$$
-b_{2,1} v_{5,0}+2 a_{3,0} v_{5,0}-b_{3,1} v_{4,0}=0
$$$$
2 b_{4,0} v_{3,2}-2 b_{2,2} v_{5,0}+2 a_{3,1} v_{5,0}=0
$$$$
2 b_{3,0} v_{3,2}-2 b_{2,2} v_{4,0}+3 a_{2,1} v_{5,0}+2 b_{4,0} v_{2,2}-2 b_{1,2} v_{5,0}+a_{3,1} v_{4,0}=0
$$$$
4 b_{4,0} v_{0,4}+a_{2,0} v_{3,2}-4 b_{0,4} v_{4,0}+3 a_{1,3} v_{4,0}-a_{3,1} v_{2,2}+5 a_{0,3} v_{5,0}+4 b_{3,0} v_{1,4}=0
$$$$
-2 b_{0,4} v_{3,2}+2 b_{2,2} v_{1,4}-2 a_{3,1} v_{1,4}+2 a_{1,3} v_{3,2}=0
$$$$
4 a_{0,3} v_{4,0}-4 b_{0,4} v_{3,0}+2 a_{1,1} v_{3,2}+4 b_{3,0} v_{0,4}+4 b_{2,0} v_{1,4}+2 a_{1,3} v_{3,0}-2 a_{3,1} v_{1,2}=0
$$$$
2 b_{2,0} v_{2,2}-2 b_{1,2} v_{3,0}+2 b_{4,0} v_{0,2}+a_{2,1} v_{3,0}-2 b_{2,2} v_{2,0}+2 b_{1,0} v_{3,2}+3 a_{1,1} v_{4,0+} 5 a_{0,1} v_{5,0}-2 b_{0,2} v_{4,0}
$$$$
+2 b_{3,0} v_{1,2}-a_{3,1} v_{2,0}=0
$$$$
4 a_{1,1} v_{5,0}+2 b_{2,0} v_{3,2}+2 a_{3,0} v_{4,0}+2 a_{3,1} v_{1,2}+2 b_{3,0} v_{2,2}-2 b_{1,2} v_{4,0}-2 b_{2,2} v_{3,0}-2 b_{0,2} v_{5,0}=0
$$$$
2 b_{2,2} v_{0,4}-a_{2,1} v_{1,4}-2 b_{0,4} v_{2,2}+2 b_{1,2} v_{1,4}-3 a_{3,1} v_{0,4}+a_{1,0} v_{2,2}+3 a_{0,3} v_{3,2}=0
$$

$3 a_{0,1} v_{3,2}+a_{1,3} v_{2,0}+a_{1,1} v_{2,2}-a_{2,1} v_{1,2}-4 b_{0,4} v_{2,0}+4 b_{1,0} v_{1,4}+4 b_{2,0} v_{0,4}+3 a_{0,3} v_{3,0}-3 a_{3,1} v_{0,2}=0$ 


$$
\begin{gathered}
4 b_{4,0} v_{1,4}+4 a_{1,3} v_{5,0}-4 b_{0,4} v_{5,0}=0 \\
2 a_{0,3} v_{2,2}+a_{0,3} v_{0,4}+2 b_{0,2} v_{1,4}-2 b_{0,4} v_{1,2}-2 a_{2,1} v_{0,4}=0 \\
b_{3,1} v_{3,2}-b_{1,3} v_{5,0}+3 a_{2,2} v_{5,0}-a_{4,0} v_{3,2}=0 \\
-b_{1,3} v_{3,2}+3 b_{3,1} v_{1,4}-a_{4,0} v_{1,4}+a_{2,2} v_{3,2}=0 \\
3 a_{1,2} v_{4,0}-a_{3,0} v_{2,2}-3 b_{1,3} v_{3,0}+b_{1,1} v_{3,2}-3 b_{0,3} v_{4,0}+b_{3,1} v_{1,2}-3 a_{4,0} v_{1,2}+a_{2,0} v_{3,2}+a_{2,2} v_{3,0+} b_{2,1} v_{2,2} \\
=0 \quad 0 \\
3 b_{2,1} v_{1,4}-2 a_{3,0} v_{1,4}-b_{0,3} v_{3,2}+3 b_{3,1} v_{0,4}-4 a_{4,0} v_{0,4}-b_{1,3} v_{2,2}+a_{1,2} v_{3,2}=0 \\
b_{1,3} v_{1,4}-a_{2,2} v_{1,4}=0 \\
-3 a_{3,0} v_{0,4}-b_{0,3} v_{2,2}+3 b_{1,1} v_{1,4}-a_{2,2} v_{1,2}+3 b_{2,1} v_{0,4}+a_{1,2} v_{2,2}-b_{1,3} v_{1,2}=0
\end{gathered}
$$

From the above system, this system must be generated

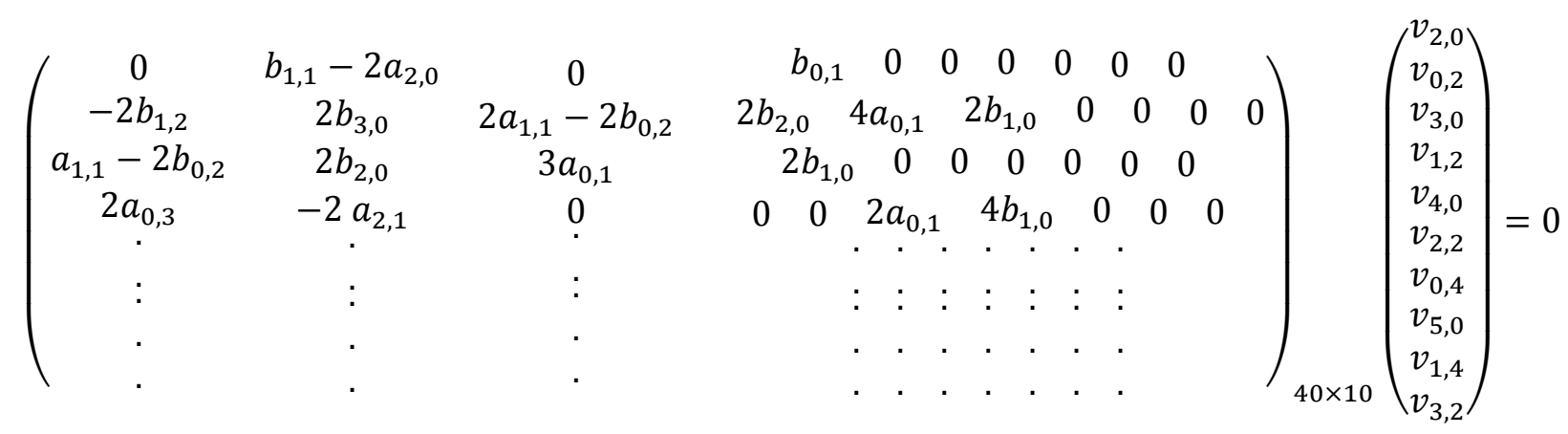

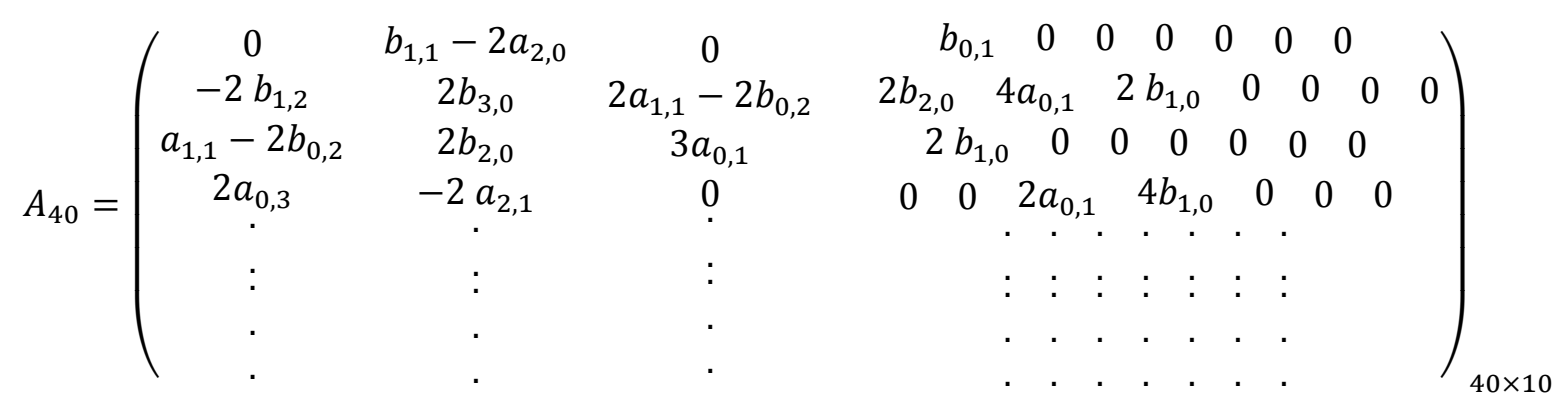

$$
\begin{aligned}
& A_{40} V^{10}=0,
\end{aligned}
$$

After solving the system, an infinite number of solutions are obtained, but the optimal solutions are as follows:

$a_{1,0}=v_{1,4}=2, a_{0,1}=v_{4,0}=-a_{1,0}, a_{2,0}=-1, a_{1,1}=-a_{2,0}, a_{3,0}=-a_{1,0}, a_{2,1}=6, a_{1,2}=v_{0,4}=$ $-4, a_{0,3}=8, a_{4,0}=b_{3,1}=v_{5,0}=a_{1,1}, a_{3,1}=-3, v_{3,2}=-a_{3,1}, a_{1,3}=a_{1,2}, a_{2,2}=b_{1,3}=a_{1,0}, b_{1,0}=$ $v_{2,0}=v_{0,2}=b_{0,1}=a_{1,0}, b_{2,0}=-\frac{3}{10}, b_{1,1}=v_{3,0}=v_{1,2}=a_{2,0}, b_{0,2}=\frac{7}{10}, b_{3,0}=b_{0,3}=a_{1,2}, b_{2,1}=$ $a_{0,1}, b_{1,2}=-a_{2,1}, b_{4,0}=\frac{13}{10}, b_{2,2}=\frac{9}{10}, b_{0,4}=-\frac{7}{5}$ and $v_{2,2}=-a_{2,1}$.

By substituting the above conditions in $V(\alpha, \beta)$, and the system (2.6), the following corollary will be our outcome:

\section{Corollary 3}

The quartic system (2.6) after substituting the above conditions with one center and one limit cycle, are based on the following system:

$$
\begin{aligned}
& \alpha=-3 \beta \alpha^{3}+6 \beta \alpha^{2}-4 \beta^{3} \alpha+8 \beta^{3}+\alpha^{4}-2 \alpha^{3} \\
& \alpha=+2 \beta^{2} \alpha^{2}-4 \beta^{2} \alpha-\alpha^{2}+2 \alpha+\beta \alpha-2 \beta \\
& \dot{\beta}=\frac{13}{10} \alpha^{4}-4 \alpha^{3}+\frac{9}{10} \beta^{2} \alpha^{2}-6 \beta^{2} \alpha-\frac{3}{10} \alpha^{2}+2 \alpha+\beta \alpha^{3} \\
& -2 \beta \alpha^{2}+2 \beta^{3} \alpha-4 \beta^{3}-\beta \alpha+2 \beta-\frac{7}{5} \beta^{4}+\frac{7}{10} \beta^{2}
\end{aligned}
$$

And the polynomial inverse integrals factor of the above system is:

$$
\begin{gathered}
V(\alpha, \beta)=2 \alpha^{2}+2 \beta^{2}-\alpha^{3}-\alpha \beta^{2}-2 \alpha^{4}-6 \alpha^{2} \beta^{2} \\
-4 \beta^{4}+\alpha^{5}+2 \alpha \beta^{4}+3 \alpha^{3} \beta^{2} .
\end{gathered}
$$

Proof: To prove this theorem by (Theorem 2) the limit cycle of system is the ellipse $\alpha^{2}+2 \beta^{2}-1=$ 0 and the center is located at the point $(3,1)$, by (Definition 3) of center.

The presence of an inverse integrals factor for Quintic polynomial systems.

\section{Theorem 7}

The nonlinear system

$\dot{\alpha}=a_{2,1} \alpha^{2} \beta+a_{0,3} \beta^{3}+a_{4,1} \alpha^{4} \beta+a_{2,3} \alpha^{2} \beta^{3}+a_{0,5} \beta^{5}$ $\dot{\beta}=b_{3,0} \alpha^{3}+b_{1,2} \beta^{2} \alpha+b_{5,0} \alpha^{5}+b_{3,2} \alpha^{3} \beta^{2}+b_{1,4} \beta^{4} \alpha$,

has an inverse integrating factor 


$$
\begin{gathered}
V(\alpha, \beta)=v_{6,0} \alpha^{6}+v_{4,2} \alpha^{4} \beta^{2}+v_{2,4} \beta^{4} \alpha^{2} \\
+v_{0,6} \beta^{6}
\end{gathered}
$$

Find the optimal solutions.

Proof: Let $V(\alpha, \beta)$ be the inverse integrating factor so by (Definition 2) and equation (1.2), Will be resulted as follows

$$
\begin{aligned}
& \mathrm{P} \frac{\partial V}{\partial \alpha}+Q \frac{\partial V}{\partial \beta}=\left(\frac{\partial \mathrm{P}}{\partial \alpha}+\frac{\partial Q}{\partial \beta}\right) V \\
&\left(a_{2,1} \alpha^{2} \beta+a_{0,3} \beta^{3}+a_{4,1} \alpha^{4} \beta+a_{2,3} \alpha^{2} \beta^{3}\right. \\
&\left.+a_{0,5} \beta^{5}\right) \frac{\partial V}{\partial \alpha} \\
&+\left(b_{3,0} \alpha^{3}+b_{1,2} \beta^{2} \alpha+b_{5,0} \alpha^{5}\right. \\
&\left.+b_{3,2} \alpha^{3} \beta^{2}+b_{1,4} \beta^{4} \alpha\right) \frac{\partial V}{\partial \beta} \\
&=\left(2 a_{2,1} \alpha \beta+4 a_{4,1} \alpha^{3} \beta\right. \\
&+2 a_{2,3} \alpha \beta^{3}+2 b_{1,2} \beta \alpha+2 b_{3,2} \alpha^{3} \beta \\
&\left.+4 b_{1,4} \beta^{3} \alpha\right) V
\end{aligned}
$$

In general, it is extremely difficult to solve quasi linear equation. Thus, the method is ignored. So by Method, $V(\alpha, \beta)=\sum_{i+j=0}^{k} V_{i, j} \alpha^{i} \beta^{j}$, and apply for $k=6$, the result will be found as follows

$$
\begin{gathered}
V(\alpha, \beta)=v_{6,0} \alpha^{6}+v_{4,2} \alpha^{4} \beta^{2}+v_{2,4} \beta^{4} \alpha^{2} \\
+v_{0,6} \beta^{6}
\end{gathered}
$$

apply equation (2.8),

$$
\begin{aligned}
& \left(\begin{array}{cccc}
4 a_{2,1}-2 b_{1,2} & 2 b_{3,0} & 0 & 0 \\
6 a_{0,3} & 2 a_{2,1} & 4 b_{3,0} & 0 \\
0 & 4 a_{0,3} & 2 b_{1,2} & 6 b_{3,0} \\
0 & 0 & 2 a_{0,3} & 4 b_{1,2}-2 a_{2,1} \\
2 a_{4,1}-2 b_{3,2} & 2 b_{5,0} & 0 & 0 \\
-4 b_{1,4}+4 a_{2,3} & 0 & 4 b_{5,0} & 0 \\
6 a_{0,5} & 2 a_{2,3}-2 b_{1,4} & 2 b_{3,2}-2 a_{4,1} & 6 b_{5,0} \\
0 & 4 a_{0,5} & 0 & -4 a_{4,1}+4 b_{3,2} \\
0 & 0 & 2 a_{0,5} & -2 a_{2,3}+2 b_{1,4}
\end{array}\right)_{9 \times 4}\left(\begin{array}{l}
v_{6,0} \\
v_{4,2} \\
v_{2,4} \\
v_{0,6}
\end{array}\right)=0 \\
& A_{9}=\left(\begin{array}{cccc}
4 a_{2,1}-2 b_{1,2} & 2 b_{3,0} & 0 & 0 \\
6 a_{0,3} & 2 a_{2,1} & 4 b_{3,0} & 0 \\
0 & 4 a_{0,3} & 2 b_{1,2} & 6 b_{3,0} \\
0 & 0 & 2 a_{0,3} & 4 b_{1,2}-2 a_{2,1} \\
2 a_{4,1}-2 b_{3,2} & 2 b_{5,0} & 0 & 0 \\
-4 b_{1,4}+4 a_{2,3} & 0 & 4 b_{5,0} & 0 \\
6 a_{0,5} & 2 a_{2,3}-2 b_{1,4} & 2 b_{3,2}-2 a_{4,1} & 6 b_{5,0} \\
0 & 4 a_{0,5} & 0 & -4 a_{4,1}+4 b_{3,2} \\
0 & 0 & 2 a_{0,5} & -2 a_{2,3}+2 b_{1,4}
\end{array}\right)_{9 \times 4}
\end{aligned}
$$

After solving the above system, the infinite numbers of solutions are obtained but the optimal solutions from the following conditions are:

$$
\begin{gathered}
a_{2,1}=a_{2,3}=-2, a_{0,3}=a_{0,5}=a_{4,1}=-1, \\
b_{3,0}=\mathrm{b}_{5,0}=b_{1,4}=-a_{2,1}, b_{1,2}=-a_{4,1}
\end{gathered}
$$

$$
\begin{aligned}
\left(a_{2,1} \alpha^{2} \beta+\right. & a_{0,3} \beta^{3}+a_{4,1} \alpha^{4} \beta+a_{2,3} \alpha^{2} \beta^{3} \\
& \left.+a_{0,5} \beta^{5}\right)\left(6 v_{6,0} \alpha^{5}+4 v_{4,1} \alpha^{3} \beta^{2}\right. \\
& \left.+2 v_{2,4} \beta^{4} \alpha\right) \\
& +\left(b_{3,0} \alpha^{3}+b_{1,2} \beta^{2} \alpha+b_{5,0} \alpha^{5}\right. \\
& +b_{3,2} \alpha^{3} \beta^{2} \\
& \left.+b_{1,4} \beta^{4} \alpha\right)\left(2 v_{4,2} \alpha^{4} \beta\right. \\
& \left.+4 v_{2,4} \beta^{3} \alpha^{2}+6 v_{0,6} \beta^{5}\right) \\
& =\left(2 a_{2,1} \alpha \beta+4 a_{4,1} \alpha^{3} \beta\right. \\
& +2 a_{2,3} \alpha \beta^{3}+2 b_{1,2} \beta \alpha+2 b_{3,2} \alpha^{3} \beta \\
& \left.+4 b_{1,4} \beta^{3} \alpha\right)\left(v_{6,0} \alpha^{6}+v_{4,2} \alpha^{4} \beta^{2}\right. \\
& \left.+v_{2,4} \beta^{4} \alpha^{2}+v_{0,6} \beta^{6}\right)
\end{aligned}
$$

After calculating, the coefficients of terms are:

$$
\begin{gathered}
\left(4 a_{2,1}-2 b_{1,2}\right) v_{6,0}+2 b_{3,0} v_{4,2}=0 \\
6 a_{0,3} v_{6,0}+2 a_{2,1} v_{4,2}+4 b_{3,0} v_{2,4}=0 \\
4 a_{0,3} v_{4,2}+2 b_{1,2} v_{2,4}+6 b_{3,0} v_{0,6}=0 \\
2 a_{0,3} v_{2,4}+\left(4 b_{1,2}-2 a_{2,1}\right) v_{0,6}=0 \\
\left(2 a_{4,1}-2 b_{3,2}\right) v_{6,0}+2 b_{5,0} v_{4,2}=0 \\
\left(-4 b_{1,4}+4 a_{2,3}\right) v_{6,0}+4 b_{5,0} v_{2,4}=0 \\
6 a_{0,5} v_{6,0}+\left(2 a_{2,3}-2 b_{1,4}\right) v_{4,2} \\
+\left(2 b_{3,2}-2 a_{4,1}\right) v_{2,4}+6 b_{5,0} v_{0,6} \\
=0 \\
4 a_{0,5} v_{4,2}+\left(-4 a_{4,1}+4 b_{3,2}\right) v_{0,6}=0 \\
2 a_{0,5} v_{2,4}+\left(-2 a_{2,3}+2 b_{1,4}\right) v_{0,6}=0
\end{gathered}
$$

the above system is given us the following 


\section{Application}

Substituting the above conditions in $V(\alpha, \beta)=$ $\sum_{i+j=0}^{k} V_{i, j} \alpha^{i} \beta^{j}$, and also to the system (2.7),these results will be obtained:

by applying the (Theorem 7) to the Quintic system

$$
\begin{gathered}
\dot{\alpha}=-2 \alpha^{2} \beta-\beta^{3}-\alpha^{4} \beta-2 \alpha^{2} \beta^{3}-\beta^{5}, \\
\dot{\beta}=2 \alpha^{3}+\beta^{2} \alpha+2 \alpha^{5}+4 \alpha^{3} \beta^{2}+2 \beta^{4} \alpha,
\end{gathered}
$$

Hence the polynomial inverse integrating factors of the above system is:

$$
V(\alpha, \beta)=v_{0,6}\left(2 \alpha^{6}+5 \alpha^{4} \beta^{2}+4 \beta^{4} \alpha^{2}+\beta^{6}\right) .
$$

this application gives us the center and also has no limit cycle.

To prove the above result, (Theorem 2) must be used, and since the inverse integrating factor is homogeneous function, then the center is located at the point $(0,0)$, by (Definition 3 ) of the center.

\section{Conclusions:}

\section{Conclusion must be based on the following points:}

1) Finding a polynomial inverse integrals factor for some systems like system (1).

2) All theories of cubic and Quintic systems do not have boundary cycles by (Theorem 2).

3 ) If a non-zero homo polynomials are the inverse integrate factor of the system (1), then it has no limit cycle.

4) For the quartic system, one center and one limit cycle are found and also one center of Quintic system is concluded.

\section{Authors' declaration:}

- Conflicts of Interest: We have no conflicts of interest to disclose.

- We hereby confirm that all the Figures and Tables in the manuscript are mine ours. Besides, the Figures and images, which are not mine ours, have been given the permission for re-publication attached with the manuscript.

- Ethical Clearance: The project was approved by the local ethical committee in University of Duhok.

\section{References:}

1. Andronov AA. Theory of bifurcations of dynamic systems on a plane. John Wiley \& Sons; 1973.

2. Ferragut A. Polynomial inverse integrating factors of quadratic differential systems and other results. Universitat Autònoma de Barcelona,; 2006.

3. García IA, Giné J, Sorolla J. On the existence of polynomial inverse integrating factors in quadratic systems with limit cycles. Dyn. Contin. Discrete Impuls. Syst. Ser. A Math. Anal. 2005 Apr 1;12:15173.

4. Giné J, Llibre J. A family of isochronous foci with Darboux first integral. Pac J Math.2005 Feb 1;218(2):343-55.

5. Giné J, Llibre J. Integrability and algebraic limit cycles for polynomial differential systems with homogeneous nonlinearities. J Differ Equ. $2004 \mathrm{Feb}$ 10;197(1):147-61.

6. García IA, Grau M. A survey on the inverse integrating factor. QTDS. 2010 Nov 1;9(1-2):115-66.

7. Coll B, Ferragut A, Llibre J. Polynomial inverse integrating factors for quadratic differential systems. Nonlinear Anal Theory Methods Appl. 2010 Aug 15;73(4):881-914.

8. Perko L. Differential equations and dynamical systems. Springer Science \& Business Media; 2013 Nov 21.

9. Poincaré H. Mémoire sur les courbes définies par une équation différentielle (I). J Math Pures Appl. 1881;7:375-422.

10. Van der Pol B. LXXXVIII. On "relaxationoscillations". Lond.Edinb.Dubl.Phil.Mag. 1926 Nov 1;2(11):978-92.

11. Yan-Qian Y. Theory of limit cycles. Translations of mathematical monographs, Vol. 66. American Mathematical Society, Providence, RI. 1986:415.

12. Hussien AM, Amen AI. On Existence Inverse Integrating Factors and First Integrals for Cubic in 2D Autonomous Differential System. ZJPAS. 2018 Jun 12;30(3):56-72.

13. Ginoux JM, Petitgirard L. Poincare's forgotten conferences on wireless telegraphy. IJBC. 2010 Nov;20(11):3617-26.

14. Giné J. On some open problems in planar differential systems and Hilbert's 16th problem. Chaos Solitons Fractals. 2007 Mar 1;31(5):1118-34.

15. Gine J. Non-existence of limit cycles for planar vector fields. Electronic J Differ Equ. 2014, vol. 2014, núm. 75, p. 1-8. 2014.

16. Laura-Guarachi L, Osuna O, Villasenor-Aguilar G. Non-existence of limit cycles via inverse integrating factors. Electron. J. Differ. Equ. 2011 Nov 8;2011(124):1-6.

17. Hilbert D. Mathematical problems. Bull New Ser Am Math Soc. 1902;8(10):437-79. 
وجود مفكوك التكامل لمعكوس متعدد الحدود ودراسات حول الدورات المنتهية لأنظمة متعددة الحدود التكعيبية والرباعية والخماسية ودول

صادق طه عبد العزيز2

احمد محمد حسين

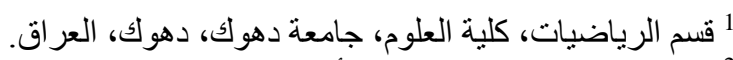

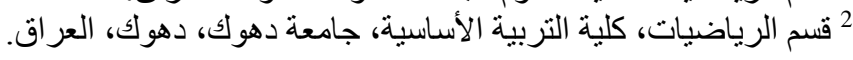

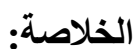

تنتمثل الأهداف الرئيسبة لدر استنا في وجود مفكوك التكامل لمعكوس متعددة الحدود ودر اسات حول الدور ات المنتهية لمتعددة الحدود

الحقيقية في نظام مستقل ثنائي الأبعاد للثنكل لأنقاف

$$
\begin{aligned}
& \dot{\alpha}=\sum_{j=0}^{m} \sum_{i=0}^{j} a_{i, j-i} \alpha^{i} \beta^{j-i}=P_{m}(\alpha, \beta) \\
& \dot{\beta}=\sum_{j=0}^{m} \sum_{i=0}^{j} b_{i, j-i} \alpha^{i} \beta^{j-i}=Q_{m}(\alpha, \beta)
\end{aligned}
$$

حيث (m) الكلمات المفتاحية: دورات منتهية ، مفكوك التكامل المعكوس ، أنظمة متعددة الحدود. 